\title{
Feynman graphs and Hyperplane arrangements defined over $\mathbb{F}_{1}$
}

\author{
Kyosuke Higashida*, Masahiko Yoshinaga ${ }^{\dagger}$
}

August 31, 2021

\begin{abstract}
Motivated by some computations of Feynman integrals and certain conjectures on mixed Tate motives, Bejleri and Marcolli posed questions about the $\mathbb{F}_{1}$-structure (in the sense of torification) on the complement of a hyperplane arrangement, especially for an arrangement defined in the space of cycles of a graph.

In this paper, we prove that an arrangement has an $\mathbb{F}_{1}$-structure if and only if it is Boolean. We also prove that the arrangement in the cycle space of a graph is Boolean if and only if the cycle space has a basis consisting of cycles such that any two of them do not share edges.
\end{abstract}

Keywords: Hyperplane arrangements, graphs, torifications.

\section{Introduction}

Theoretical physics, especially quantum field theory and Feynman integrals, raises many mathematical problems [1]. In [2], Bejleri and Marcolli studied certain algebraic varieties associated with Feynman integrals for graphs from the viewpoint of mixed Tate motives and Grothendieck ring of varieties. They also discuss the $\mathbb{F}_{1}$-structure (torification, see [3]) of these varieties, which is a more recent perspective.

The category of mixed Tate motives is conjectured to be generated by the objects defined by using hyperplane arrangements. Therefore, it is a natural question to ask whether a hyperplane arrangement has an $\mathbb{F}_{1}$-structure or not. In [2], a combinatorial necessary condition for an arrangement to have $\mathbb{F}_{1}$-structure was given.

The purpose of this paper is to answer questions posed in [2, Question 5.3, 5.4]. Namely, we discuss the $\mathbb{F}_{1}$-structure on the complement of a hyperplane arrangement, especially for an arrangement defined in the cycle space of a graph which is closely related to a variety appearing in the Feynman integral [2, §2.2, §2.3].

The plan of the present paper is as follows. In \$2, we recall some basic notions of hyperplane arrangements, especially, the notions of the characteristic polynomial and the Boolean arrangement. In $\$ 3$, we show that an arrangement has an $\mathbb{F}_{1}$-structure if and only if it is Boolean. We also show that, under an additional assumption, the combinatorial necessary condition formulated in [2] is sufficient for the $\mathbb{F}_{1}$-structure. In $\$ 4$, we consider the arrangement defined by a graph $([2$, $\S 2.3 .2]$ ). We prove that the arrangement is Boolean (namely having $\mathbb{F}_{1}$-structure) if and only if the first homology group of the original graph has a basis consisting of simple cycles which do not share edges.

${ }^{*}$ Graduate School of Mathematical Science, The University of Tokyo, 3-8-1 Komaba, Meguroku, Tokyo, 1538914, JAPAN. E-mail: k-higashida@g.ecc.u-tokyo.ac.jp

$\dagger$ (Corresponding author) Department of Mathematics, Faculty of Science, Hokkaido University, North 10, West 8, Kita-ku, Sapporo 060-0810, JAPAN E-mail: yoshinaga@math.sci.hokudai.ac.jp 


\section{Characteristic polynomial}

In this section, we recall some basic notions of hyperplane arrangements. See 4 for details. Let $\mathcal{A}=\left\{H_{1}, \ldots, H_{n}\right\}$ be an arrangement of affine hyperplanes in a vector space $V$ with $\operatorname{dim} V=\ell$ over a field $\mathbb{K}$. The intersection poset is the set $L(\mathcal{A})=\{\cap S \mid S \subset \mathcal{A}$ with $\cap S \neq \emptyset\}$ of nonempty intersections of $\mathcal{A}$. The intersection poset $L(\mathcal{A})$ is partially ordered by reverse inclusion, which has a unique minimal element $\widehat{0}=V$. The arrangement $\mathcal{A}$ is said to be central if $L(\mathcal{A})$ has a unique maximal element $\cap \mathcal{A} \neq \emptyset$, and is said to be essential if the maximal elements of $L(\mathcal{A})$ are 0 dimensional subspaces. An arrangement $\mathcal{A}$ is called a Boolean arrangement if $L(\mathcal{A})$ is isomorphic to a Boolean lattice, i.e., the lattice of all subsets of a ground set. Let $\mathcal{A}=\left\{H_{1}, \ldots, H_{n}\right\}$ be a Boolean arrangement. Then there exists a system of coordinates $\left(x_{1}, \ldots, x_{\ell}\right)$ of $V$ such that $H_{i}$ is equal to the coordinate hyperplane $\left\{x_{i}=0\right\}$ for $1 \leq i \leq n$. In particular, we have $n \leq \ell$. An arrangement $\mathcal{A}$ is the essential Boolean arrangement if and only if $\mathcal{A}$ is essential and $n=\ell$.

Next, we recall the definition of the characteristic polynomial $\chi(\mathcal{A}, t)$. The Möbius function $\mu: L(\mathcal{A}) \longrightarrow \mathbb{Z}$ is defined by

$$
\mu(X)= \begin{cases}1, & \text { if } X=V, \\ -\sum_{V \leq Y<X} \mu(Y), & \text { if } X>V .\end{cases}
$$

Then $\chi(\mathcal{A}, t)=\sum_{X \in L(\mathcal{A})} \mu(X) t^{\operatorname{dim} X}$. One of the most important properties of $\chi(\mathcal{A}, t)$ is the following deletion-restriction formula ([4, Cor. 2.57]). Let us fix a hyperplane $H \in \mathcal{A}$. Then naturally the deletion $\mathcal{A}^{\prime}:=\mathcal{A} \backslash\{H\}$ and restriction $\mathcal{A}^{\prime \prime}:=H \cap \mathcal{A}^{\prime}$ are defined. Note that $\mathcal{A}^{\prime \prime}$ is a reduced arrangement in the space $H$. Then the following recursive formula holds.

$$
\chi(\mathcal{A}, t)=\chi\left(\mathcal{A}^{\prime}, t\right)-\chi\left(\mathcal{A}^{\prime \prime}, t\right) .
$$

Define the complement of $\mathcal{A}$ by $M(\mathcal{A})=V \backslash \bigcup_{H \in \mathcal{A}} H$. It is easily seen that $M\left(\mathcal{A}^{\prime}\right)=M(\mathcal{A}) \sqcup$ $M\left(\mathcal{A}^{\prime \prime}\right)$. Therefore in the Grothendieck ring $K_{0}\left(\mathcal{V} a r_{\mathbb{K}}\right)$ of varieties over $\mathbb{K}$, we have

$$
[M(\mathcal{A})]=\chi(\mathcal{A}, \mathbb{L})
$$

where $\mathbb{L}=\left[\mathbb{A}_{\mathbb{K}}^{1}\right]$ is the class of the affine line. Suppose $\mathcal{A}$ is defined over $\mathbb{Z}$. Then, for a prime power $q=p^{r}$ with $p \gg 0$, the intersection poset $L(\mathcal{A})$ is isomorphic to $L\left(\mathcal{A} \otimes \mathbb{F}_{q}\right)$. Therefore, $\left|M\left(\mathcal{A} \otimes \mathbb{F}_{q}\right)\right|=\chi(\mathcal{A}, q)$.

Example 2.1. Let $\mathcal{A}$ be the Boolean arrangement defined by $x_{1} x_{2} \ldots x_{n}=0$ in $\mathbb{K}^{\ell}(n \leq \ell)$. Then $\chi(\mathcal{A}, t)=(t-1)^{n} t^{\ell-n}$.

Remark 2.2. Let $\mathcal{A}$ be an arrangement in $\mathbb{K}^{\ell}$ which is not necessarily central. Then the coning $c \mathcal{A}$ ([4, Definition 1.15]) is a central arrangement in $\mathbb{K}^{\ell+1}$. We have $M(c \mathcal{A})=M(\mathcal{A}) \times \mathbb{K}^{\times}$. So from now on, we assume that all arrangements are central.

\section{Arrangements with torified complements}

A torification ([2, 3] ) of a scheme $X$ over $\mathbb{Z}$ is a morphism of schemes $e: T \longrightarrow X$, such that $T=\bigsqcup_{j} \mathbb{G}_{m}^{d_{j}}$ is a disjoint union of split tori (where $G_{m}=\operatorname{Spec} \mathbb{Z}\left[t, t^{-1}\right]$ ), the restriction $\left.e\right|_{\mathbb{G}_{m}^{d_{j}}}$ is an isomorphism into a locally closed subscheme of $X$, and $e(\mathbb{K}): T(\mathbb{K}) \longrightarrow X(\mathbb{K})$ is bijective for every field $\mathbb{K}$.

Suppose that

(a) $\mathcal{A}$ is the Boolean arrangement.

Then, there exists a coordinate system such that $\mathcal{A}$ is defined as $\left\{x_{1}=0\right\}, \ldots,\left\{x_{n}=0\right\}$ in $\mathbb{K}^{\ell}$ and $M(\mathcal{A}) \simeq\left(\mathbb{K}^{\times}\right)^{n} \times \mathbb{K}^{\ell-n}$. This implies (see [3, §1.3.2]) 
(b) $M(\mathcal{A})$ has a torification, more precisely, there exists a torified scheme $X$ such that $X \otimes \mathbb{K}$ is isomorphic to $M(\mathcal{A})$ as varieties over $\mathbb{K}$.

Suppose that $M(\mathcal{A})$ has a torification. Then in the Grothendieck ring, $[M(\mathcal{A})]$ is expressed as a linear combination of $(\mathbb{L}-1)^{i}, i \geq 0$, with nonnegative coefficients. Therefore, we have the following.

(c) Suppose $\chi(\mathcal{A}, t)=\sum_{i} c_{i} \cdot(t-1)^{i}$ is the Taylor expansion of $\chi(\mathcal{A}, t)$ at $t=1$. Then $c_{i} \geq 0$ for all $i \geq 0$.

Then, $(a) \Longrightarrow(b) \Longrightarrow(c)$ hold $([2])$. Now we discuss other implications.

Lemma 3.1. Let $\mathcal{A}=\left\{H_{1}, \ldots, H_{n}\right\}$ be an arrangement in $V=\mathbb{K}^{\ell}$. If $(c)$ holds, then $n \leq \ell$.

Proof. Recall that the $\chi(\mathcal{A}, t)$ is a polynomial of the form $t^{\ell}-n t^{\ell-1}+($ terms of $\operatorname{deg} \leq \ell-2)$. It is equal to $(t-1)^{\ell}+(\ell-n)(t-1)^{\ell-1}+($ terms of deg $\leq \ell-2)$. Hence $(c)$ implies $\ell-n \geq 0$.

Theorem 3.2. Let $\mathcal{A}=\left\{H_{1}, \ldots, H_{n}\right\}$ be an arrangement in $\mathbb{K}^{\ell}$. Then,

(1) (a) and (b) are equivalent.

(2) Suppose $\mathcal{A}$ is essential. Then (a), (b) and (c) are equivalent.

Proof. We first consider (2). If $\mathcal{A}$ is essential, then by definition, $n \geq \ell$. Suppose $(c)$ holds. Then Lemma 3.1 tells that $n \leq \ell$, hence $n=\ell$, which implies $(a)$.

Next we consider $(1)$. We need to prove $(b) \Longrightarrow(a)$. Let us assume that $M(\mathcal{A})$ has a torification. Then there exists a dominant morphism $\left(\mathbb{K}^{\times}\right)^{\ell} \hookrightarrow M(\mathcal{A})$. Suppose that $\mathcal{A}$ is not Boolean. Then there exist dependent hyperplanes. Namely, after a change of coordinates, we may assume $\left\{x_{1}=0\right\}, \ldots,\left\{x_{r}=0\right\},\left\{x_{1}+x_{2}+\cdots+x_{r}=0\right\}$ (with $2 \leq r \leq n-1$ ) are in $\mathcal{A}$. Then we have $\left(\mathbb{K}^{\times}\right)^{\ell} \hookrightarrow M(\mathcal{A}) \subset\left\{x_{1} x_{2} \ldots x_{r}\left(x_{1}+\cdots+x_{r}\right) \neq 0\right\} \subset \mathbb{K}^{\ell}$. Taking the ring of functions, we have an embedding of $\mathbb{K}$-algebras.

$$
\mathbb{K}\left[x_{1}^{ \pm 1}, \ldots, x_{r}^{ \pm 1}, x_{r+1}, \ldots, x_{\ell}, \frac{1}{x_{1}+\cdots+x_{r}}\right] \subset \mathbb{K}\left[t_{1}^{ \pm 1}, \ldots, t_{\ell}^{ \pm 1}\right] .
$$

However, this is impossible. Because in the right hand side, the set of invertible elements is equal to the set of monomials, which are linearly independent over $\mathbb{K}$. However, in the left hand side, $x_{1}, \ldots, x_{r}, x_{1}+\cdots+x_{r}$ are dependent invertible elements.

The following example shows that if $\mathcal{A}$ is not essential, $(c)$ is not sufficient for $(a)$ or $(b)$.

Example 3.3. Consider the arrangement $\mathcal{A}$ defined by $x_{1} x_{2} x_{3}\left(x_{1}+x_{2}+x_{3}\right)=0$ in $\mathbb{K}^{4}$. Then, $\chi(\mathcal{A}, t)=t^{4}-4 t^{3}+6 t^{2}-3 t=(t-1)^{4}+(t-1)$. Hence the coefficients of $(t-1)^{i}$ are nonnegative, however, $\mathcal{A}$ is not Boolean.

\section{The arrangement in the cycle space of a graph}

Let $\Gamma=(V, E)$ be a finite graph. Let $H_{1}(\Gamma)=H_{1}(\Gamma, \mathbb{K})$ be the cycle space over $\mathbb{K}$.

Since $\Gamma$ is a 1-dimensional CW-complex, any 1-cochain is automatically a cocycle. Therefore, any edge $e \in E$ (equipped with an orientation) determines an element of $H^{1}(\Gamma)=\operatorname{Hom}\left(H_{1}(\Gamma), \mathbb{K}\right)$. We denote the element by $\eta_{e}: H_{1}(\Gamma) \longrightarrow \mathbb{K}$.

If $\eta_{e} \neq 0$, then $H_{e}:=\operatorname{Ker} \eta_{e}$ defines a hyperplane in $H_{1}(\Gamma) \simeq \mathbb{K}^{b_{1}(\Gamma)}$. As in [2, define $\mathcal{A}_{\Gamma}$ as $\mathcal{A}_{\Gamma}:=\left\{H_{e} \mid e \in E, \eta_{e} \neq 0\right\}$. We note that $e, e^{\prime} \in E$ with $e \neq e^{\prime}$ may define the same hyperplane. We just forget the multiplicities and consider the reduced arrangement.

Lemma 4.1. The arrangement $\mathcal{A}_{\Gamma}$ is essential. In particular, $\left|\mathcal{A}_{\Gamma}\right| \geq b_{1}(\Gamma)$. 
Proof. Since $\{e \in E\}$ spans the space of 1-cochains, $\left\{\eta_{e}\right\}$ generates the space of 1-cocycles, hence $H^{1}(\Gamma)$. We have $\cap_{e \in E} H_{e}=\{0\}$.

Let $\mathcal{F}$ be a spanning forest of $\Gamma$. Let $e \in E \backslash \mathcal{F}$. Then the vertices of $e$ are connected by the unique minimal path in $\mathcal{F}$. By adding $e$ to this path, we have a simple cycle $C_{e} \subset E$. By putting suitable orientations, the set of such cycles $\mathcal{B}(\mathcal{F}):=\left\{C_{e} \subset E \mid e \in E \backslash \mathcal{F}\right\}$ forms a basis of $H_{1}(\Gamma)$. Such a basis is called a fundamental basis [6].

Definition 4.2. Let $\mathcal{B} \subset H_{1}(\Gamma)$ be a basis consisting of simple cycles (that is, each cycle passes an edge at most once). Then $\mathcal{B}$ is said to be separated if any two cycles $C, C^{\prime} \in \mathcal{B}\left(C \neq C^{\prime}\right)$ do not share edges.

Theorem 4.3. Let $\Gamma=(V, E)$ be a graph. Then the following are equivalent.

(i) $M\left(\mathcal{A}_{\Gamma}\right)$ has a torification.

(ii) $\mathcal{A}_{\Gamma}$ is Boolean.

(iii) $\left|\mathcal{A}_{\Gamma}\right|=b_{1}(\Gamma)$

(iv) For any spanning forest $\mathcal{F} \subset \Gamma$, the fundamental basis $\mathcal{B}(\mathcal{F})$ is separated.

(v) There exists a spanning forest $\mathcal{F} \subset \Gamma$ such that the fundamental basis $\mathcal{B}(\mathcal{F})$ is separated.

(vi) There exists a separated basis $\mathcal{B}$.

Proof. $(i) \Longleftrightarrow($ ii $) \Longleftrightarrow($ iii $)$ is obtained from Theorem 3.2 and Lemma 4.1. Also (iv) $\Longrightarrow$ $(v) \Longrightarrow(v i)$ is obvious. Now we assume $(v i)$. Let $\mathcal{B}=\left\{C_{1}, \ldots, C_{b}\right\}$ be a basis as in (vi). Then every spanning forest is obtained from $\Gamma$ by removing an edge of $C_{i}$ for each $i$. Thus $\mathcal{B}$ is the fundamental basis for every spanning forest $\mathcal{F}$. This proves $(v i) \Longrightarrow(i v)$.

Now we prove $(v i) \Longrightarrow(i i)$. Let $\mathcal{F}$ be a basis as in $(v i)$. Then every edge $e \in E$ is either contained in the unique cycle $C \in \mathcal{B}$ or not contained in $\bigcup_{C \in \mathcal{B}} C$. In the latter case, $\eta_{e}=0$, hence we do not need to consider. Hence $\left\{\eta_{e}\right\} \subset H^{1}(\Gamma)$ forms a dual basis of $\mathcal{B}$. Thus $\mathcal{A}_{\Gamma}$ is Boolean.

Finally, we prove $(i i) \Longrightarrow(i v)$. Suppose $(i i)$ and there exists a spanning forest $\mathcal{F}$ such that $\mathcal{B}(\mathcal{F})$ is not separated. There exists an edges $e_{0} \in E$ such that $\left\{C \in \mathcal{B}(\mathcal{F}) \mid C \ni e_{0}\right\}$ contains more than one cycles. Let $E \backslash \mathcal{F}=\left\{e_{1}, \ldots, e_{b}\right\}$. Then $\left\{H_{e_{0}}, H_{e_{1}}, \ldots, H_{e_{b}}\right\} \subset \mathcal{A}_{\Gamma}$ forms $b+1$ distinct hyperplanes. This contradicts the fact that $\mathcal{A}_{\Gamma}$ is Boolean. This completes the proof.

Remark 4.4. The graph satisfying the conditions in Theorem 4.3 is obtained by finitely many repetitions of gluing a graph with $b_{1} \leq 1$ at a vertex.

Remark 4.5. There is another way to associate a hyperplane arrangement $\mathcal{A}(\Gamma)$ to a graph $\Gamma$, which is called the graphic arrangement (see [4, §2.4] for details). The two arrangements $\mathcal{A}_{\Gamma}$ and $\mathcal{A}(\Gamma)$ are dual to each other in the sense of matroids (see [5]). In view of this interpretation, Theorem 4.3 characterizes those graphs $\Gamma$ for which the simplification of the dual of the circuit matroid of $\Gamma$ is the free matroid.

Acknowledgements. Masahiko Yoshinaga was partially supported by JSPS KAKENHI Grant Numbers JP19K21826, JP18H01115. The authors thank the referee for pointing out Remark 4.5. 


\section{References}

[1] S. Bloch, H. Esnault, D. Kreimer, On motives associated to graph polynomials. Comm. Math. Phys. 267 (2006), no. 1, 181-225.

[2] D. Bejleri, M. Marcolli, Quantum field theory over $\mathbb{F}_{1}$. J. Geom. Phys. 69 (2013), 40-59.

[3] J. López Peña, O. Lorscheid, Torified varieties and their geometries over $\mathbb{F}_{1}$. Math. Z. 267 (2011), no. 3-4, 605-643.

[4] P. Orlik, H. Terao, Arrangements of Hyperplanes. Grundlehren Math. Wiss. 300, SpringerVerlag, New York, 1992.

[5] J. Oxley, Matroid theory. Second edition. Oxford Graduate Texts in Mathematics, 21. Oxford University Press, Oxford, 2011.

[6] Sysło, M. M. On cycle bases of a graph. Networks 9 (1979), no. 2, 123-132. 